$\underline{\text { Supplementary Information }}$

\title{
Interface induced high-performance piezoelectric nanogenerator based on electrospun 3-phase composite nanofiber for wearable applications
}

\author{
Sakti Prasanna Muduli ${ }^{1}$, Sushmitha Veeralingam ${ }^{1}$, Sushmee Badhulika ${ }^{1 *}$ \\ ${ }^{1}$ Department of Electrical Engineering, Indian Institute of Technology Hyderabad, Hyderabad 502285, India \\ *Corresponding author E-mail address:sbadh@iith.ac.in
}

\section{Content}

\begin{tabular}{|c|c|}
\hline Section & Title \\
\hline S1 & XRD and Raman Spectrum of $\mathrm{MoS}_{2}$ \\
\hline S2 & Composition Analysis \\
\hline S3 & Measurement of force applied by finger tapping \\
\hline S4 & Mechanical vibration frequency measurement \\
\hline S5 & Cost per device estimation \\
\hline
\end{tabular}

\section{S1. XRD and Raman Spectrum of $\mathrm{MoS}_{2}$}

XRD pattern and Raman spectrum of as synthesized $\mathrm{MoS}_{2}$ is given in Figure S1 and S2 respectively. The prominent peaks of the $\mathrm{MoS}_{2}$ are present at $2 \theta$ values of $14.5^{\circ}, 34^{\circ}, 38^{\circ}$ and $59^{\circ}$ which can be indexed according to JCPDS card number 37-1492. In the Raman spectrum, the well known $\mathrm{E}^{1}{ }_{2 \mathrm{~g}}$ and $A_{1 \mathrm{~g}}$ peaks are seen at around Raman shift of $381 \mathrm{~cm}^{-1}$ and $406 \mathrm{~cm}^{-1}$ which correspond to in-plane and out-of-plane vibration of S atom with respect to Mo atom respectively. ${ }^{1}$ The difference in Raman shift of $25 \mathrm{~cm}^{-1}$ shows that, as synthesized $\mathrm{MoS}_{2}$ sheets may consist of 6 - 7 layers. ${ }^{1}$ 


\section{$\underline{\text { Supplementary Information }}$}
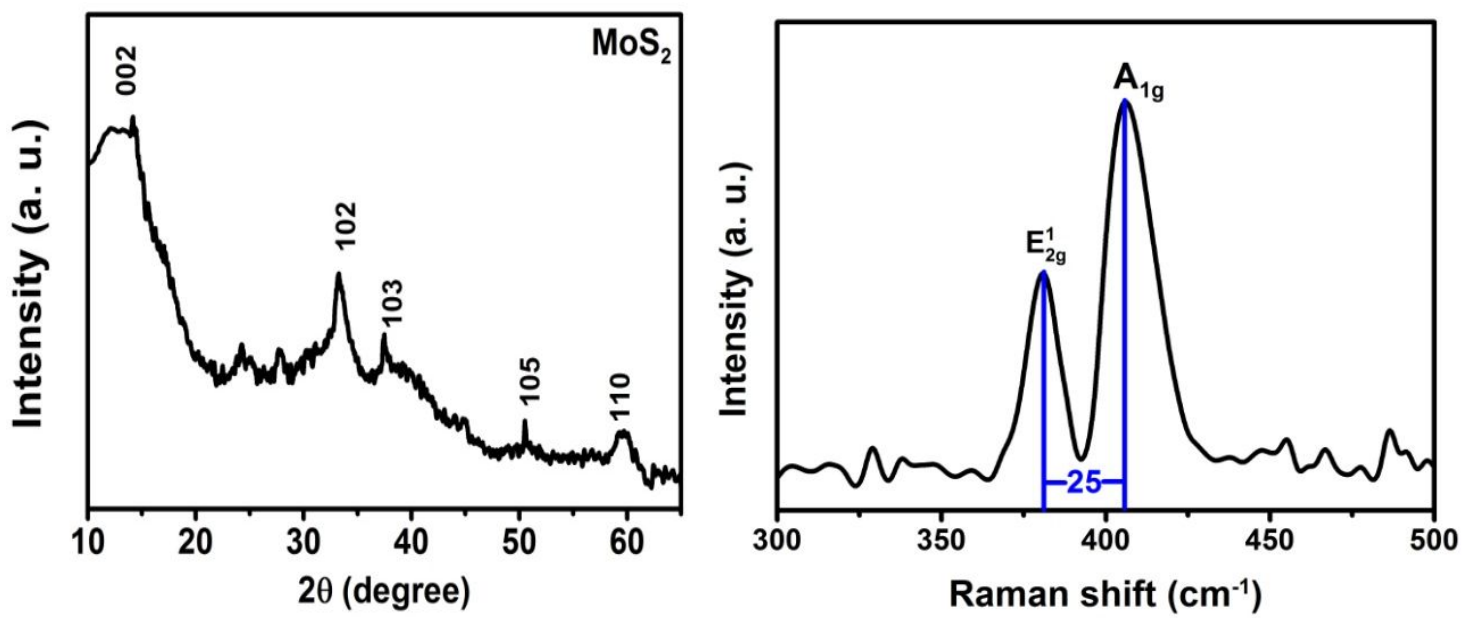

Figure S1: XRD pattern of $\mathrm{MoS}_{2}, \mathrm{S2}$ : Raman spectra of $\mathrm{MoS}_{2}$

\section{S2. Composition Analysis}

Energy Dispersive X-Ray Analysis (EDAX) patterns of composite nanofiber mats is depicted in Figure S2 and the measured weight percentage of all the elements present in the composite nanofiber are listed in Table S1. Presence of all the claimed elements can be observed from the respective peaks and the variation of $\mathrm{MoS}_{2}$ concentration can be verified from the measured elemental weight percentage.
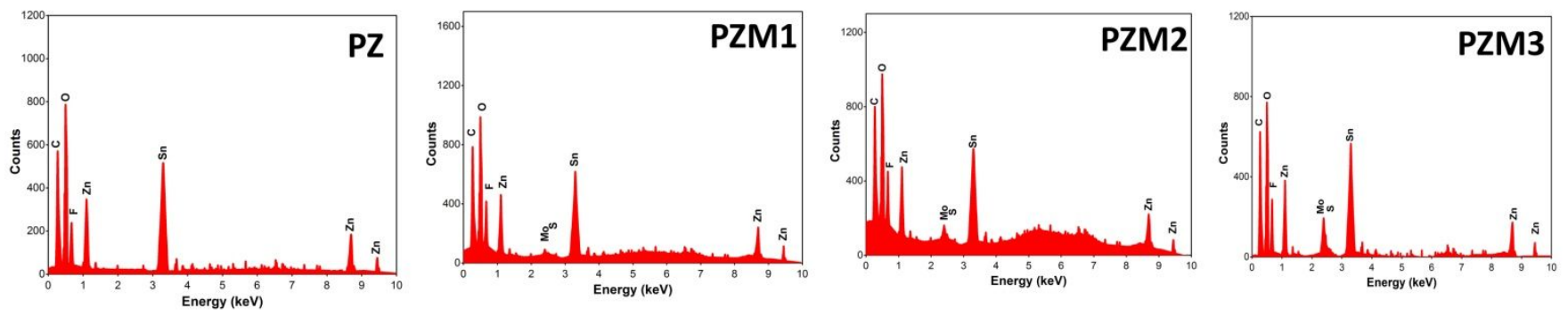

Figure S2 EDAX composition analysis of the composite nanofiber mat

Table: S1 Weight percentage of the element measured by EDAX

\begin{tabular}{cccccccc}
\hline $\begin{array}{c}\text { Elements } \rightarrow \\
\text { Samples } \downarrow\end{array}$ & C & O & F & Zn & Sn & Mo & S \\
\hline PZ & 29.79 & 9.17 & 37.75 & 8.51 & 14.78 & 0 & 0 \\
\hline PZM1 & 30.33 & 9.30 & 36.63 & 7.64 & 13.65 & 0.93 & 0.52 \\
\hline PZM2 & 30.02 & 9.74 & 36.28 & 7.12 & 12.57 & 2.53 & 1.65 \\
\hline PZM3 & 29.58 & 9.27 & 35.67 & 6.92 & 11.85 & 3.98 & 2.73 \\
\hline
\end{tabular}




\section{$\underline{\text { Supplementary Information }}$}

\section{S3. Measurement of force applied by Finger tapping}

Figure S3 (a) represents a simple arrangement for measurement of applied force by finger tapping. In detail, the PENG is placed on a high precision weight scale to measure the hand tapping force in terms of weight $(\mathrm{kg})$ so that the hand tapping force can be approximately controlled with this feedback. The finger impart involves the physical model combining the gravity and pulse term. ${ }^{2}$ When a mass (here finger) imparts on another thin object (film), there are two processes involved: (1) initially touching the surface, and (2) completely acting on the thin object. The decreasing velocity of the imparting mass increases and reaches to minimum value in the first process and gradually nullifies in the second process. Hence, based on the kinetic energy and momentum theorem, the following equation can be framed.

$$
\begin{gathered}
\boldsymbol{m} \boldsymbol{g} \boldsymbol{h}=\frac{\mathbf{1}}{\mathbf{2}} \boldsymbol{m} \boldsymbol{v}^{\mathbf{2}} \ldots \ldots \ldots \ldots \ldots \ldots \ldots \ldots \text { Equation }(\mathrm{S} 1) \\
(\boldsymbol{F}-\boldsymbol{m} \boldsymbol{g}) \Delta \boldsymbol{t}=\boldsymbol{m} \boldsymbol{v} \ldots \ldots \ldots \ldots \ldots \ldots \text { Equation }(\mathrm{S} 2) \\
\boldsymbol{\sigma}=\frac{\boldsymbol{F}}{\boldsymbol{s}} \ldots \ldots \ldots \ldots \ldots \ldots \text { Equation }(\mathrm{S} 3)
\end{gathered}
$$

Where $\boldsymbol{m}$ is the mass of the imparting object, $\boldsymbol{h}$ is the height of falling, $\boldsymbol{v}$ is the maximum velocity of falling, $\boldsymbol{\sigma}$ is the pressure developed during contact or applied stress, $\boldsymbol{F}$ is the contact force, $\boldsymbol{s}$ is the contact area, and $\Delta t$ is the time spanning during second process.

In this case $\mathrm{m}=\sim 1 \mathrm{~kg}$ (Finger tapping force measured weight scale)

$\mathrm{S}=4 \times 10^{-4} \mathrm{~m}^{2}$ (effective area of the PENG)

$\Delta t=0.33$ second (for $3 \mathrm{~Hz}$ frequency)

$\mathrm{H}=0.1 \mathrm{~m}$

$\mathrm{g}=9.8 \mathrm{~m} \mathrm{~s}^{-2}$

This gives the contact force of $\sim 10 \mathrm{~N}$ and this gives the pressure $\sim 25 \mathrm{kPa}$. 


\section{Supplementary Information}

The voltage output of the PZM2 with three different forces and at the same $3 \mathrm{~Hz}$ frequency is depicted in Figure S3 (b). The average $\mathrm{V}_{\mathrm{oc}}$ was found to be $5 \mathrm{~V}, 16 \mathrm{~V}$ and $26 \mathrm{~V}$ for soft tapping, medium tapping and hard tapping respectively. Above $1 \mathrm{kgf}$ force, the voltage output got saturated so the test was conducted up to $1 \mathrm{kgf}$ only. The voltage output of a piezoelectric device is generally represented as Equation $\mathrm{S} 4 .^{3}$

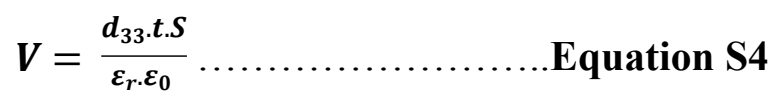

Where $\mathrm{V}$ is the voltage output, $\mathrm{d}_{33}$ is the piezoelectric strain constant in 33 direction, $\mathrm{t}$ is the thickness of the piezoelectric material, $\mathrm{S}$ is the applied mechanical stress (in Pascal) $\varepsilon_{\mathrm{r}}$ and $\varepsilon_{\mathrm{o}}$ represent the relative permittivity of piezoelectric material and permittivity of free space. From this equation, it is clear that output voltage is directly proportional to the stress applied on the PENG. But this is valid in the linear portion transfer characteristic of a piezoelectric device because, after the saturation of voltage output, higher stress does not causes higher voltage. ${ }^{3}$ When higher stress is applied on a piezoelectric material, more number of dipole forming unit cells contributes to the voltage output whereas with a gradual increment of mechanical stress, after a threshold amount of force, there will be no more dipole forming unit cells in the material to contribute to the energy conversion and results in saturation of $\mathrm{V}_{\mathrm{oc}}$.
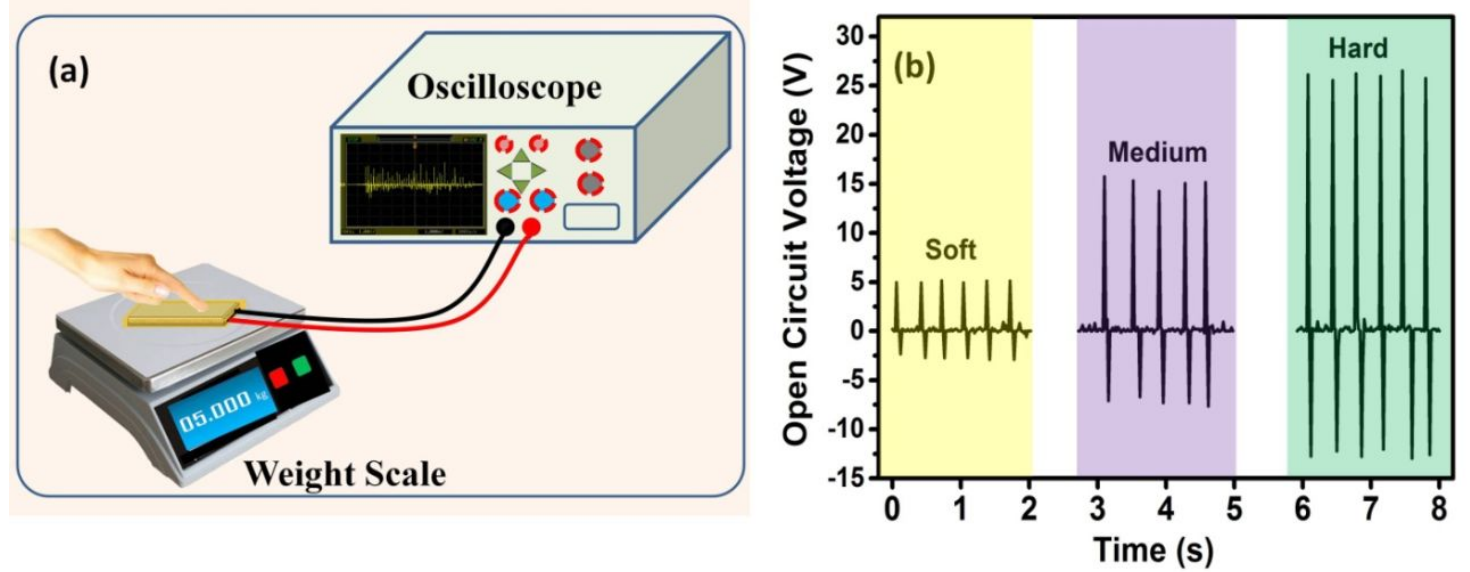

Figure S3 (a) Arrangement for measurement of force applied by hand tapping; (b) $\mathrm{V}_{\text {oc }}$ depending on different levels of finger tapping for PZM2 based PENG

\section{S4. Mechanical Vibration Frequency Measurement}

Further, the PENG was kept under the base of a vacuum pump (Rockyvac 400, Tarson India, weight of $5.2 \mathrm{~kg}$ ) for vibration energy harvesting but the voltage output was very low $(0.4 \mathrm{~V})$. The 


\section{Supplementary Information}

voltage signal is shown in Figure S4. During analysis of the signal, it was found that the signal is having around 22 peaks (either positive or negative) per second. In a minute it is 1320, which is almost nearer to the rotational speed of the pump (1400 rpm). So this PENG may be used for mechanical vibration frequency measurement.

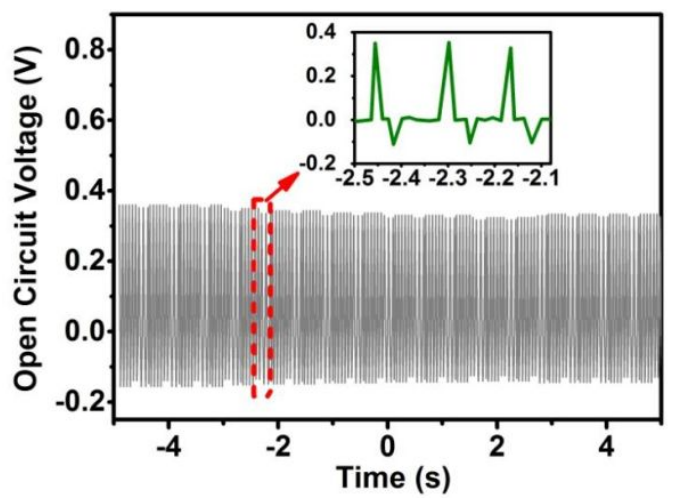

Figure S4 Voltage signal from PENG when placed under a mechanical vibrating device

\section{S5: Detailed calculation of cost per PVDF-ZSO-MoS ${ }_{2}$ nanofiber mat-based PENG}

\section{Cost of chemicals per device}

1. PVDF pellets $(16 \mathrm{wt} \%):-\$ 0.15$

2. $\operatorname{DMF}(2 \mathrm{ml}):-\$ 0.045$

3. Acetone $(1 \mathrm{ml}):-\$ 0.011$

4. Zinc acetate dihydrate $(0.66 \mathrm{~g}):-\$ 0.175$

5. Sodium stannate trihydrate $(0.8 \mathrm{~g}):-\$ 0.221$

6. Sodium molybdate $(0.3 \mathrm{~g}):-\$ 0.154$

7. Thiourea $(0.6 \mathrm{~g}):-\$ 0.127$

Cost of insulating material and electrodes

8. ITO coated PET substrate $(2 \mathrm{~cm} \times 2 \mathrm{~cm}):-\$ 0.132$

9. Polymide tape $(2 \mathrm{~cm} \times 10 \mathrm{~cm}):-\$ 0.012$

Cost per PENG device: - Cost of chemicals used + cost of electrodes and insulating material $=$ $\$ 1.027$ 


\section{Supplementary Information}

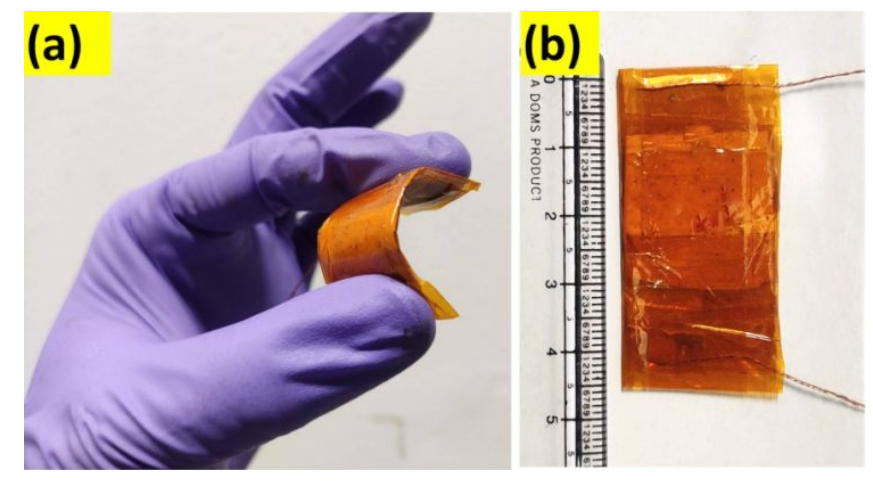

Figure S5 (a) Flexibility of PENG, (b) image of PENG

\section{References}

(1) Liang, L., \& Meunier, V. (2014). First-principles Raman spectra of $\mathrm{MoS}_{2}, \mathrm{WS}_{2}$ and their heterostructures. Nanoscale, 6(10), 5394-5401. https://doi.org/10.1039/C3NR06906K

(2) Hou, C.; Huang, T.; Wang, H.; Yu, H.; Zhang, Q.; Li, Y. A strong and stretchable self healing film with self-activated pressure sensitivity for potential artificial skin applications. Sci. Rep. 2013, 3, 3138. https://doi.org/10.1038/srep03138

(3) KocK B., Uchino k. (2000), Piezoelectric Ultrasonic Motors https://doi.org/10.1016/B0-08$\underline{042993-9 / 00161-3}$ 